\title{
Artenschutz im Fokus der höchstrichterlichen Rechtsprechung
}

\author{
Eine Analyse der Jahre 2014 bis 2019
}

\section{Martin Kment*}

(c) Der/die Autor(en) 2020

Die Bedeutung des Artenschutzrechts nimmt rasant zu. Industrieanlagen, Infrastrukturprojekte oder sonstige größere Bauvorhaben lassen sich kaum mehr ohne eine genauere Studie des Artenschutzrechts in Angriff nehmen, will man nicht unerwünschte Überraschungen in der Realisierungsphase erleben. Dabei reicht es häufig nicht aus, nur die gesetzlichen Vorgaben zu studieren. Auch die einschlägige Rechtsprechung bedarf einer eingehenden Betrachtung. Dieser letztgenannten Aufgabe nimmt sich der vorliegende Beitrag an. Er analysiert die höchstgerichtliche Rechtsprechung zum Artenschutzrecht, destilliert deren Kernaussagen und ordnet die Judikate systematisch den Grundstrukturen des Artenschutzrechts zu. Betrachtungszeitraum sind die Jahre 2014 bis einschließlich 2019.

\section{Einleitung}

Das Artenschutzrecht war in den vergangenen Jahren verstärkt Gegenstand höchstrichterlicher Entscheidungen, die offene Rechtsfragen geklärt und das Rechtsgebiet stetig weiterentwickelt haben. Die meisten Judikate bezogen sich au die artenschutzrechtlichen Zugriffsverbote aus $\$ 44$ BNatSchG und die naturschutzfachliche Einschätzungsprärogative, da diesen Rechtsfeldern in der behördlichen und gerichtlichen Praxis die größte Beachtung geschenkt wird. Überragende Bedeutung nimmt hier der Beschluss des BVerfG vom 23.10.2018 ein, der die weitere Entwicklung des Artenschutzrechts in den nächsten Jahren wesentlich prägen wird. ${ }^{1}$ Andere Entscheidungen befassten sich mit der artenschutzrechtlichen Ausnahme, mit den Schutz- und Vermeidungsmaßnahmen bezüglich der artenschutzrechtlichen Zugriffsverbote oder mit allgemeinen Rechtsfragen zum Artenschutz im Verwaltungs- und Rechtsschutzverfahren.

Die nachfolgende Untersuchung möchte die Entwicklung des Artenschutzrechts in der höchstrichterlichen Rechtsprechung der letzten fünf Jahre darstellen. Sie beschränkt sich - auch bei den Quellennachweisen - im Wesentlichen auf Urteile und Beschlüsse des BVerwG, bezieht aber auch andere Entscheidungen, u.a. des BVerfG, des $\mathrm{BGH}$ und des EuGH mit ein, soweit sie sich substanziell auf das Artenschutzrecht beziehen. Aufgrund der Fülle des Materials ist die Untersuchung notwendigerweise selektiv.

\section{Allgemeine Rechtsfragen zum Artenschutz in Verwaltungs- und Rechtsschutzverfahren}

\subsection{Artenschutz und UVP-Vorprüfung}

Die Vorschriften des Artenschutzes sind wesentlicher Bestandteil von Genehmigungsverfahren für Vorhaben im Außenbereich, insbesondere bei Infrastrukturvorhaben wie

Prof. Dr. Martin Kment, LL.M (Cambridge),

Lehrstuhl für Öffentliches Recht und Europarecht, Umweltrecht und Planungsrecht

und geschäftsführender Direktor des Instituts für Umweltrecht der Universität Augsburg,

Augsburg, Deutschland
Bundesfernstraßen, Windparks oder Stromfreileitungen. Die Vorhaben, die im Genehmigungsverfahren mit den Vorschriften des Artenschutzrechts konfrontiert werden, müssen oftmals einer Vorprüfung über die Umweltverträglichkeit nach dem UVPG unterzogen werden. In diesem Kontext stellt das BVerwG klar, dass artenschutzrechtliche Belange lediglich in der allgemeinen Vorprüfung über die UVP-Pflicht nach $\$ 7$ Abs. 1 UVPG Prüfungsgegenstand sind; bei standortbezogenen Vorprüfungen nach $\$ 7$ Abs. 2 UVPG sollen die artenschutzrechtlichen Vorschriften grundsätzlich nicht zu berücksichtigen sein ${ }^{2}$. Die umfassende Prüfung der artenschutzrechtlichen Vorschriften im Genehmigungsverfahren bleibt davon allerdings unberührt.

\subsection{Ergänzendes Verfahren}

Werden die artenschutzrechtlichen Vorschriften im Genehmigungsverfahren relevant, gewinnt ihre Verbotswirkung sehr schnell eine hohe Bedeutung, insbesondere in Planfeststellungsverfahren. Teilelement der artenschutzrechtlichen Prüfung ist auch die Untersuchung der artenschutzrechtlichen Ausnahme nach $₫ 45$ Abs. 7 BNatSchG. Findet diese Untersuchung nicht statt, obwohl sie nach den Umständen des Einzelfalls angezeigt war, so ist der Rechtsverstoß im Wege des ergänzenden Verfahrens behebbar ${ }^{3}$. Selbiges gilt für den Fall, dass die Planfeststellungsbehörde Schutzmaßnahmen im Sinne von $\$ 44$ Abs. 5 S. 2 Nr. 1 BNatSchG hätte anordnen müssen, um die signifikante Erhöhung des Kollisionsrisikos besonders geschützter Arten mit dem planfestgestellten Vorhaben auszuschließen. Geeignete Schutzmaßnahmen zur Kollisionsvermeidung können ebenfalls im Wege der Planergänzung nachgeholt werden ${ }^{4}$.

\subsection{Keine subjektiv-rechtliche Qualität}

artenschutzrechtlicher Regeln, Ausgleichsanspruch nach $\$ 906$ Abs. 2 S. 2 BGB analog

Die Vorschriften des Artenschutzes dienen gem. $\$ 37$ Abs. 1 S. 1 BNatSchG (allein), ,dem Schutz der wildlebenden Tier- und Pflanzenarten" und können daher zugunsten von Individualpersonen keine Rechtsschutzposition vermitteln. Dies gilt auch gegenüber Gemeinden, auch wenn

*) Der Verfasser dankt Herrn Dennis Jurdt für die Unterstützung be der Erstellung dieser Publikation. Bei den zitierten Entscheidungen mussten zum Teil unterschiedliche Fundstellen angegeben werden, da teilweise die relevanten Passagen der Entscheidungen nur an unterschiedlichen Stellen abgedruckt wurden. Fanden sich keine Nachweise in amtlichen Sammlungen oder Fachzeitschriften, wurden die Entscheidungen nach JURIS zitiert.

1) BVerfG, Beschl. v. 23.10.2018 - 1 BvR 2523/13 und 1 BvR 595/14, NuR 2019, 33.

2) BVerwG, Urt. v. 26.9.2019 - 7 C 5.18, NuR 2020, 182, Rdnr. $31 \mathrm{ff}$

3) BVerwG, Urt. v. 8.1.2014 - 9 A 4.13, BVerwGE 149, 31, Rdnr. 81, 96.

4) BVerwG, Beschl. v. 23.1.2015 - 7 VR 6.14, NuR 2015, 257, Rdnr. 30. 
Gemeindeeigentum tangiert ist. Ein Verstoß gegen zwingende artenschutzrechtliche Vorschriften verschafft Kommunen keine wehrfähige Rechtsposition, denn die artenschutzrechtlichen Vorschriften ,sind nicht dazu bestimmt, das Grundeigentum einer Gemeinde oder das gemeindliche Selbstverwaltungsrecht zu schützen"

Die öffentlich-rechtliche Schutzrichtung des Artenschutzrechts verhindert allerdings nicht, dass Betroffene einen zivilrechtlichen Ausgleichsanspruch nach $\$ 906$ Abs. 2 S. 2 BGB analog erlangen. Dies kann etwa zugunsten von Grundstückeigentümern und sonstigen Nutzungsberechtigten eintreten, wenn artenschutzrechtlich besonders geschützte Arten wiederangesiedelt werden: Nach einer Entscheidung des BGH folgt aus $\$ 65$ Abs. 1 S. 1 BNatSchG eine Duldungspflicht im Sinne von $\$ 1004$ Abs. 2 BGB für die von der Wiederansiedlung betroffenen Grundstückseigentümer und sonstigen Nutzungsberechtigten, wenn hinter dieser naturschutzrechtlichen Maßnahme ein hinreichendes staatliches Regelungskonzept steht, das als geeignete öffentlich-rechtliche Grundlage für die naturschutzrechtliche Maßnahme dient ${ }^{6}$. Dies gilt jedenfalls, soweit die Nutzung des Grundstücks durch die naturschutzrechtliche Maßnahme nicht unzumutbar beeinträchtigt wird ${ }^{7}$. Der Duldungspflicht korrespondiert grundsätzlich die Möglichkeit, sich als Eigentümer analog $\$ 906$ Abs. 2 S. 2 BGB die Schäden ersetzen zu lassen, die durch die naturschutzrechtlichen Maßnahmen entstehen ${ }^{8}$. Dieser nachbarrechtliche Ausgleichsanspruch entfällt jedoch, sofern das Naturschutzrecht oder das Artenschutzrecht dem Störer verbieten, die Störung zu beseitigen. Das ist jedenfalls dann der Fall, wenn die Beseitigung der wiederangesiedelten besonders geschützten Arten gegen die artenschutzrechtlichen Zugriffsverbote aus $\$ 44$ BNatSchG verstoßen würde 9 .

\section{Europäische Vorgaben zum Artenschutzrecht}

Das deutsche Artenschutzrecht geht auf die Umsetzung europäischer Richtlinien zurück, die zum Ziel haben, innerhalb der Europäischen Union ein einheitlich hohes Schutzniveau für wildlebende Tier- und Pflanzenarten zu gewährleisten. Die bekanntesten Rechtsakte sind die Fauna-Flora-Habitat-Richtlinie (,FFH-Richtlinie“") ${ }^{10}$ und die Vogelschutzrichtlinie, ${ }^{11}$ die als wesentliche Vorlagen des heutigen deutschen Artenschutzrechts im BNatSchG dienten. In den vergangenen Jahren hat sich der EuGH mit Fragen zur Auslegung dieser Richtlinien und Anforderungen an die Umsetzung auseinandergesetzt.

Nach Art. 12 Abs. 1 FFH-Richtlinie haben die Mitgliedstaaten die Verpflichtung, die notwendigen Maßnahmen zu treffen, um ein strenges Schutzsystem für besonders geschützte Arten in deren natürlichen Verbreitungsgebieten einzuführen. Der EuGH hat klargestellt, dass die Mitgliedstaaten hierbei nicht nur einen vollständigen gesetzlichen Rahmen zur Etablierung eines strengen Schutzsystems schaffen müssen, sondern darüber hinaus auch konkrete Maßnahmen zum Schutz betroffener besonders geschützter Arten durchführen müssen. Ein Schutzsystem im Sinne von Art. 12 Abs. 1 FFH-Richtlinie muss imstande sein, das Eintreten der Verbotstatbestände aus Art. 12 Abs. 1 FFH-Richtlinie tatsächlich wirksam zu verhindern. ${ }^{12} \mathrm{Da}-$ her setzt ein solches strenges Schutzsystem auch den „Erlass kohärenter und koordinierter vorbeugender Maßnahmen voraus"13. Das bedeutet, dass die Mitgliedstaaten nicht nur dazu verpflichtet sind, einen vollständigen gesetzlichen Rahmen zu schaffen, um ein strenges artenschutzrechtliches Schutzsystem zu etablieren, sondern es muss national auch vorgeschrieben sein, welche konkreten Schutzmaßnahmen zugunsten besonders geschützter Arten präventiv eingeleitet werden ${ }^{14}$. Der EuGH bestätigte hiermit seine bisherige Linie $^{15} \mathrm{zu}$ den Anforderungen an eine richtlinienkonforme Umsetzung des Art. 12 Abs. 1 FFH-Richtlinie.

Von den Anforderungen an Art. 12 FFH-Richtlinie können die Mitgliedstaaten unter den Voraussetzungen des
Art. 16 FFH-Richtlinie abweichen. Der EuGH hat in einer noch jungen Entscheidung Voraussetzungen formuliert, unter denen nach Art. 16 Abs. 1 lit. e) FFH-Richtlinie von dem in Art. 12 Abs. 1 lit. a) FFH-Richtlinie manifestierten Verbot der absichtlichen Tötung abgewichen werden darf ${ }^{16}$. Dazu gehören insbesondere strenge Anforderungen an die Nachweise, die die Mitgliedstaaten hinsichtlich der mit der Ausnahme verfolgten Ziele erbringen müssen ${ }^{17}$. Grund hierfür ist, dass Art. 16 Abs. 1 lit. e) FFH-Richtlinie, anders als die anderen Ausnahmetatbestände in Art. 16 Abs. 1 FFH-Richtlinie, kein Ziel für die Ausnahme vorgibt $^{18}$. Quasi als Kompensation für diese tatbestandliche Weite ist dem Mitgliedstaat bzw. den nationalen Behörden für eine richtlinienkonforme Anwendung des Art. 16 Abs. 1 lit. e) FFH-Richtlinie abzuverlangen, die Geeignetheit und Erforderlichkeit der Ausnahme zur Zielerreichung nachzuweisen ${ }^{19}$. Durch die hohen Hürden, die der EuGH zur Abweichung von den Verboten des Art. 12 Abs. 1 lit. a) FFH-Richtlinie aufstellt, wird deutlich, dass er eine restriktive Anwendung der Abweichungsausnahme befürwortet ${ }^{20}$.

Abweichungsmöglichkeiten beschäftigten den EuGH auch hinsichtlich der Vogelschutzrichtlinie. Dort sieht Art. 5 Vogelschutzrichtlinie in Bezug auf besonders geschützte Vogelarten die Verpflichtung der Mitgliedstaaten vor, erforderliche Maßnahmen zur Schaffung einer allgemeinen Schutzregelung zu treffen. Von dieser Verpflichtung kann nach Maßgabe des Art. 9 Vogelschutzrichtlinie abgewichen werden. Um die richtlinienkonforme Abweichung für nationale Stellen zu ermöglichen, ist ,der nationale rechtliche Rahmen der Abweichungsbefugnis so zu gestalten, dass er den Grundsätzen der Rechtssicherheit entspricht"21. Dafür muss ,die nationale Regelung die Kriterien für die Abweichung klar und präzise anführen und die nationalen Stellen verpflichten, diese zu berücksichtigen“"22. Ferner soll die

5) BVerwG, Urt. v. 9.11.2017 - 3 A 2.15, Rdnr. 26 = NVwZ 2018, Beilage 1, 51 Rdnr. 26.

6) BGH, Urt. v. 19.7.2019 - V ZR 177/17, MDR 2019, 1444, Rdnr. 35, 45

7) BGH, Urt. v. 19.7.2019 - V ZR 177/17, MDR 2019, 1444, Rdnr. 31

8) BGH, Urt. v. 19.7.2019 - V ZR 177/17, MDR 2019, 1444, Rdnr. 67

9) BGH, Urt. v. 19.7.2019 - V ZR 177/17, MDR 2019, 1444, Rdnr. $67 \mathrm{f}$

10) Richtlinie 92/43/EWG des Rates v. 21.5.1992 zur Erhaltung der natürlichen Lebensräume sowie der wildlebenden Tiere und Pflanzen, AB1. L 206, S. 7, zuletzt geändert durch Richtlinie 2006/105/EG des Rates v. 20.11.2006, ABl. L 363, S. 368.

11) Richtlinie 2009/147/EG des europäischen Parlaments und des Rates vom 30.11.2009 über die Erhaltung der wildlebenden Vogelarten, AB1. L 20, S. 7.

12) EuGH, Urt. v. 17.4.2018 - C-441/17, ECLI:EU:C:2018:255, ZUR 2018, 349 Rdnr. 231 - Kommission/Polen.

13) EuGH, Urt. v. 17.4.2018 - C-441/17, ECLI:EU:C:2018:255, ZUR 2018, 349 Rdnr. 231 - Kommission/Polen.

14) EuGH, Urt. v. 10.11.2016 - C-504/14, ECLI:EU:C:2016:847, Rdnr. 140 - Kommission/Griechenland.

15) So auch zuvor EuGH, Urt. v. 15.3.2012 - C-340/10, ECLI:EU: C:2012:143, NuR 2012, 259, Rdnr. 60 f. - Kommission/Zypern; EuGH, Urt. v. 9.6.2011 - C-383/09, ECLI:EU:C:2011:369, NuR 2011, 498, Rdnr. 19 ff. - Kommission/Frankreich.

16) EuGH, Urt. v. 10.10.2019 - C-674/17, ECLI:EU:C:2019:851, NuR 2019, 756, Rdnr. 80.

17) EuGH, Urt. v. 10.10.2019 - C-674/17, ECLI:EU:C:2019:851, NuR 2019, 756, Rdnr. 41.

18) EuGH, Urt. v. 10.10.2019 - C-674/17, ECLI:EU:C:2019:851, NuR 2019, 756, Rdnr. 34.

19) EuGH, Urt. v. 10.10.2019 - C-674/17, ECLI:EU:C:2019:851, NuR 2019, 756, Rdnr. 45, 47, 49, 80.

20) EuGH, Urt. v. 10.10.2019 - C-674/17, ECLI:EU:C:2019:851, NuR 2019, 756, Rdnr. 30.

21) EuGH, Urt. v. 21.6.2018 - C-557/15, ECLI:EU:C:2018:477, NuR 2018, 477, Rdnr. 47 - Kommission/Malta.

22) EuGH, Urt. v. 21.6.2018 - C-557/15, ECLI:EU:C:2018:477, NuR 2018, 477, Rdnr. 47 - Kommission/Malta. 
Abweichung von den nationalen Stellen nur mit angemessener Begründung erfolgen dürfen und auf Ausnahmefälle beschränkt sein ${ }^{23}$. Die Mitgliedstaaten müssen bei der Genehmigung von Abweichungen nach Art. 9 Vogelschutzrichtlinie zahlreiche Gesichtspunkte berücksichtigen, so unter anderem geographische, klimatische oder biologische ${ }^{24}$.

\section{Entscheidungen zu den artenschutzrechtlichen Zugriffsverboten aus $₫ 44$ Abs. 1 BNatSchG}

4.1. Das Tötungsverbot gemäß SS 44 Abs. $1 \mathrm{Nr} .1$, Abs. 5 S. 2 Nr. 1 BNatSch G

4.1.1 Der Begriff der Signifikanz, $\int 44$ Abs. 5 S. 2 Nr. 1 BNatSchG

\subsubsection{Entwicklung des Signifikanzkriteriums,} unionsrechtliche Unbedenklichkeit

Das artenschutzrechtliche Tötungsverbot aus $\int \$ 44$ Abs. 1 Nr. 1, Abs. 5 S. 2 Nr. 1 BNatSchG ist als Teil der artenschutzrechtlichen Zugriffsverbote nach $\$ 6$ Abs. 1 Nr. 2 BImSchG im immissionsschutzrechtlichen Genehmigungsverfahren und gem. $₫ 35$ Abs. $3 \mathrm{Nr}$. 5 BauGB im Baugenehmigungsverfahren von besonderer Relevanz für Infrastrukturvorhaben wie Windparks, Stromtrassen oder Bundesautobahnen. Es ist als ordnungsrechtliche Schutzvorschrift ausgestaltet und stellt einen Verbotstatbestand dar, der einem genehmigungsbedürftigen Vorhaben entgegenstehen kann. Seine Schutzwirkung umfasst nach $\$ \$ 7$ Abs. 2 Nr. 13, Nr. 14 BNatSchG insbesondere die in Anhang IV der FFH-Richtlinie und die in Anlage 1 Bundesartenschutzverordnung (BArtSchV) aufgelisteten besonders geschützten und streng geschützten Arten.

Ihren Ursprung finden die artenschutzrechtlichen Zugriffsverbote im Europarecht, insbesondere in der FFH-Richtlinie und der Vogelschutzrichtlinie. Sie sind nach europäischer Vorstellung in ihrer Anwendung tatbestandlich durch ein subjektives Element eingeschränkt. So regelt Art. 12 Abs. 1 lit. a) der FFH-Richtlinie, dass ,alle absichtlichen Formen des Tötens“ untersagt seien. Der deutsche Gesetzgeber hatte in nationaler Umsetzung dieser Richtlinie auf ein subjektives Element verzichtet, sodass es in $\$ 42$ Abs. 1 Nr. 1 BNatSchG a.F. (2007) verboten war, ,wildlebende Tiere der besonders geschützten Arten [...] zu töten“. Um den so entstandenen zu weiten Tatbestand der deutschen Umsetzung des artenschutzrechtlichen Tötungsverbots in $\$ 42$ Abs. 1 Nr. 1 BNatSchG a.F. (2007) einzuschränken, entwickelte das BVerwG das verengende Tatbestandsmerkmal der ,signifikanten Erhöhung des Tötungsrisikos“ für Individuen besonders und streng geschützter Arten im Vorhabenraum. ${ }^{25}$ Überdies versteht das BVerwG das Tötungsverbot aus $\$ 44$ Abs. 1 Nr. 1 BNatSchG seit jeher als individuenbezogen ${ }^{26}$, sodass populationsbezogene Auswirkungen der Tötung nur eingeschränkt zu beachten sind.

Der Gesetzgeber hat die korrigierende Rechtsprechung aufgegriffen und den Begriff der ,,signifikanten Erhöhung des Tötungsrisikos" durch das Gesetz zur Änderung des Bundesnaturschutzgesetzes vom 15.9.201727 in $\$ 44$ Abs. 5 S. 2 Nr. 1 BNatSchG n. F. implementiert ${ }^{28}$. Flankierend bestätigt das BVerwG in ständiger Rechtsprechung die Vereinbarkeit des selbst entwickelten Signifikanzkriteriums mit dem Europarecht. ${ }^{29}$ Es meint, dass ohne eine signifikante Erhöhung des Tötungsrisikos ,nicht davon gesprochen werden kann, dass eine Tötung im Sinne der europäischen Richtlinien bewusst in Kauf genommen wird" 30 . Das Signifikanzkriterium sei dazu gedacht, ,,unverhältnismäßige Folgen für die Zulassung von Vorhaben bei der Anwendung des artenschutzrechtlichen Tötungsverbots zu vermeiden“"31. Ohne das Signifikanzkriterium wären Vorhaben im Außenbereich, ,nur noch unter Anwendung von Ausnahmetatbeständen möglich, und die europarechtlich vorgesehene Ausnahme würde zur Regel“"32.

\subsubsection{Grundrisiko für Tiere}

In der Sache hat das BVerwG mit seiner Auffassung zum Signifikanzkriterium Recht, denn ohne das einschränkende Tatbestandsmerkmal der ,signifikanten Erhöhung des Tötungsrisikos" wäre rechtmäßiges Verwaltungshandeln ohne die latente Gefahr von Tötungen einzelner Tiere durch ein Bauvorhaben nie völlig ausgeschlossen ${ }^{33}$. Führt man sich vor Augen, dass tierisches Leben nicht in einer unberührten $\mathrm{Na}$ tur existiert, sondern in einer vom Menschen geschaffenen und beeinflussten Landschaft, birgt diese Nutzung durch den Menschen nämlich stets „ein spezifisches Grundrisiko für

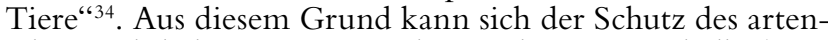
schutzrechtlichen Tötungsrisikos auch nur innerhalb dieses Rahmens bewegen ${ }^{35}$, sodass nicht jede Tötung eines Individuums den Verbotstatbestand des $\$ 44$ Abs. 1 Nr. 1 BNatSchG verwirklichen kann. Vielmehr liegt ein Verstoß gegen das artenschutzrechtliche Tötungsverbot nur dann vor, wenn das Risiko einer Tötung dasjenige allgemeine Risiko übersteigt, welches mit einem Vorhaben dieser Art ,als gewöhnlichem Bestandteil des Naturraums immer verbunden ist“" ${ }^{\text {"36 }}$ Ein Tötungsrisiko kann daher dem Grunde nach hinzunehmen sein, ,wenn es zwar von Menschen verursacht ist, aber nur einzelne Individuen betrifft" ${ }^{\text {"37 }}$. Hieraus lässt sich ableiten, dass die Signifikanzprüfung kein Nullrisiko fordert ${ }^{38}$.

\subsubsection{3 Überschreiten des alloemeinen Risikos}

Das beschriebene allgemeine Risiko wird überschritten, wenn besondere Umstände hinzutreten, welche dazu führen, dass durch die Realisierung des geplanten Vorhabens von einer signifikanten, also hinreichend qualifizierten Gefährdung für Individuen besonders geschützter Arten gesprochen werden $\mathrm{kann}^{39}$. Wann solche besonderen Umstände beim geplanten Vorhaben vorliegen, ist eine Frage wertender Ge-

23) EuGH, Urt. v. 21.6.2018 - C-557/15, ECLI:EU:C:2018:477, NuR 2018, 477, Rdnr. 47 - Kommission/Malta.

24) EuGH, Urt. v. 21.6.2018 - C-557/15, ECLI:EU:C:2018:477, NuR 2018, 477, Rdnr. $62-$ Kommission/Malta.

25) BVerwG, Urt. v. 12.3.2008 - 9 A 3/06, BVerwGE 130, 299 Rdnr. 219f; BVerwG, Urt. v. 9.7.2009 - 4 C 12/07, BVerwGE 134, 166, Rdnr. 42.

26) BVerwG, Urt. v. 9.2.2017-7 A 2.15, BVerwGE 158, 1, Rdnr. 466

27) BGBl. 2017 I, S. 3434.

28) BVerwG, Urt. v. 27.11.2018 - 9 A 8.17, BVerwGE 163, 380 , Rdnr. 99; vgl. auch die Begründung zum Gesetzesentwurf des Gesetzes zur Änderung des BNatSchG v. 12.4.2017, BTDrs. 18/11939, S. 17.

29) BVerwG, Urt. v. 27.11.2018 - 9 A 8.17, BVerwGE 163, 380, Rdnr. 97.

30) BVerwG, Urt. v. 27.11.2018 - 9 A 8.17, BVerwGE 163, 380 , Rdnr. 101.

31) BVerwG, Urt. v. 27.11.2018 - 9 A 8.17, BVerwGE 163, 380, Rdnr. 101.

32) BVerwG, Urt. v. 27.11.2018 - 9 A 8.17, BVerwGE 163, 380, Rdnr. 101.

33) BVerwG, Urt. v. 9.2.2017 - 7 A 2.15, BVerwGE 158, 1, Rdnr. 466.

34) BVerwG, Urt. v. 10.11.2016 - 9 A 18.15, BVerwGE 156, 215, Rdnr. 83; ähnlich BVerwG, Urt. v. 27.11.2018 - 9 A 8.17, BVerwGE 163, 380, Rdnr. 98.

35) BVerwG, Urt. v. 10.11.2016 - 9 A 18.15, BVerwGE 156, 215 , Rdnr. 83; BVerwG, Urt. v. 6.4.2017 - 4 A 16.16, NuR 2018, 255, Rdnr. 74

36) BVerwG, Urt. v. 10.11.2016 - 9 A 18.15, BVerwGE 156, 215 Rdnr. 83

37) BVerwG, Urt. v. 10.11.2016 - 9 A 18.15, BVerwGE 156, 215 , Rdnr. 83; BVerwG, Urt. v. 6.4.2017 - 4 A 16.16, NuR 2018, 255, Rdnr. 74.

38) BVerwG, Urt. v. 28.4.2016 - 9 A 9.15, BVerwGE 155, 91, Rdnr. 141.

39) BVerwG, Urt. v. 9.2.2017 - 7 A 2.15, BVerwGE 158, 1, Rdnr. 466; BVerwG, Urt. v. 27.11.2018 - 9 A 8.17, BVerwGE 163, 380, Rdnr. 98. 
samtbetrachtung im Einzelfall ${ }^{40}$. In diese Gesamtbetrachtung sind auch Schadensvermeidungsmaßnahmen einzustellen, deren Umsetzung dazu führen kann, dass „das baubedingte Tötungsrisiko durch Vermeidungsmaßnahmen bis zur Schwelle des allgemeinen Lebensrisikos, dem die Individuen der jeweiligen Art ohnehin unterliegen, gesenkt wird“41.

Weiter kann von einem Übersteigen des allgemeinen Risikos hin zu einer signifikanten Gefährdung von betroffenen Individuen der besonders geschützten Arten grundsätzlich nur dann gesprochen werden, wenn sich betriebsbedingte Risiken oder bau- und anlagenbezogene Risiken verwirklichen ${ }^{42}$. Es muss also um „Individuen solcher besonders geschützter Arten (gehen), die aufgrund ihrer Verhaltensweise gerade im Bereich des Vorhabens ungewöhnlich stark von dessen Risiken betroffen sind und diese Risiken sich durch die konkrete Umsetzung des Vorhabens einschließlich der geplanten Maßnahmen zur Vermeidung oder Verminderung von Kollisionen oder sonstiger Tötungen nicht beherrschen lassen" "43.

Ferner setzt die Beurteilung eines Übersteigens des allgemeinen Lebensrisikos eine Vielzahl langwieriger Untersuchungen des Raums voraus, in welchem sich das geplante Vorhaben befinden soll. In der behördlichen Praxis werden hierzu regelmäßig sog. „Raumnutzungsanalysen“ vorgenommen. Dies setzt zwingend eine ausreichende Ermittlung und Bestandsaufnahme der im Einwirkungsbereich des Vorhabens vorhandenen Tierarten und ihrer vorhandenen Lebensräume voraus. „Das verpflichtet die Behörde (aber) nicht, ein lückenloses Arteninventar zu erstellen, die Untersuchungstiefe hängt vielmehr maßgeblich von den naturräumlichen Gegebenheiten im Einzelfall $\mathrm{ab}^{44}$.

\subsubsection{Merkmale „besonderer Umstände“}

Um der Praxis bei der Anwendung des $₫ 44$ Abs. 5 S. 2 Nr. 1 BNatSchG behilflich zu sein, hat das BVerwG das Merkmal der ,,besonderen Umstände“" präzisiert: Als besondere Umstände, die für die Beurteilung der Signifikanz eine Rolle spielen, kommen insbesondere artspezifische Verhaltensweisen, die Häufigkeit der Frequentierung des vom Vorhaben durchschnittenen Raums und die Wirksamkeit vorgesehener Schutzmaßnahmen, die das erhöhte Tötungsrisiko senken sollen, in Betracht ${ }^{45}$. Darüber hinaus können noch weitere Kriterien bedeutsam sein, die „,im Zusammenhang mit der Biologie der Art" stehen ${ }^{46}$.

Gerade mit dieser letztgenannten, eher offenen Formulierung (,,im Zusammenhang mit der Biologie der Art“) verdeutlicht das Gericht die denkbare Relevanz einer Vielzahl an möglichen Kriterien, wodurch auch weiterhin die praktische Umsetzung des artenschutzrechtlichen Tötungsverbots besondere Schwierigkeiten bereitet. Auf Seiten der Behörden sind hierdurch weitgehende Freiheiten entstanden, selbst Kriterien für die Beurteilung der signifikanten Erhöhung des Tötungsverbots zu bilden und zur Bewertung der praktischen Sachlage im Einzelfall heranzuziehen.

Hierdurch ist eine Behördenpraxis begünstigt worden, die zu gesteigerter Unsicherheit für Vorhabenträger geführt hat. Deshalb wurden - insbesondere von den einzelnen Ländern - praxistaugliche Anwendungshilfen in Form von Artenschutzleitfäden und verschiedenen Erlassen für Behörden erstellt, um auf eine einheitliche Behördenpraxis hinzuwirken. ${ }^{47}$ Daneben treten Handlungsempfehlungen und Indizes von Umweltverbänden, Naturschutzorganisationen und Wissenschaftlern, die sich zum Ziel nehmen, die allgemeinen Kriterien des BVerwG naturschutzfachlich zu würdigen. Allerdings erweist sich die Fachwissenschaft im Bereich des Artenschutzes nicht immer als eindeutiger Erkenntnisgeber, da diese durch eine Vielzahl an wissenschaftlichen Streitfragen geprägt ist ${ }^{48}$. Das BVerwG sah in dieser Entwicklung den ausdrücklichen Willen des Gesetzgebers bestätigt, dass Konzepte, die die verallgemeinerten Kriterien der Rechtsprechung für den konkreten Fall ausfüllen und damit als Werkzeug zur Bestimmung der signifikanten Erhöhung des Tötungsrisikos dienen, praxisbezogen weiterentwickelt wer- den ${ }^{49}$. Dazu zählt das BVerwG ${ }^{50}$ beispielsweise den „,Mortalitäts-Gefährdungs-Index wildlebender Vögel im Rahmen von Projekten und Eingriffen" von Dierschke \& Bernotat"

Wo Auskünfte über die geschützten Arten fehlen, entsteht zwangsläufig ein Entscheidungsspielraum der zuständigen Behörde, der mangels wissenschaftlicher Erkenntnisse gerichtlich nicht voll überprüft werden kann. Das Gericht ist darauf zurückgeworfen zu prüfen, „ob das Vorgehen der Behörde im konkreten Einzelfall naturschutzfachlich vertretbar ist und nicht etwa auf einem unzulänglichen oder gar ungeeigneten Verfahren beruht."52 Dieser exekutive Freiraum schrumpft in dem Maße, in dem sich ein fachlich anerkannter Standard bildet ${ }^{53}$. Darüber hinaus ist die Behörde verpflichtet, den ,,allgemeinen maßgeblichen Standard der ,besten einschlägigen wissenschaftlichen Erkenntnisse ${ }^{6}$ einzuhalten (...), was die ,Ausschöpfung aller wissenschaftlichen Mittel und Quellen' (...) voraussetzt" ${ }^{\text {"54 }}$. Im Fall von Fledermäusen bedeutet dies etwa, dass die zuständige Behörde nur dann von einem Übersteigen des allgemeinen Tötungsrisikos ausgehen darf, ,wenn Hauptflugrouten oder bevorzugte Jagdgebiete durch das geplante Vorhaben betroffen sind“"55.

4.1.2 Zurückgenommene gerichtliche Kontrolle und die Abkehr von der naturschutzfachlichen Einschätzungsprärogative

\subsubsection{Auswirkungen abstrakter Rechtsbegriffe und fachlicher Erkenntnisdefizite}

Bei der fachlichen Bewertung, ob das Tötungsrisiko im konkreten Fall dasjenige allgemeine Lebensrisiko übersteigt, welchem die Individuen besonders geschützter Arten im menschlich gestalteten Naturraum ohnehin ausgesetzt sind, kommen

40) BVerwG, Urt. v. 10.11.2016 - 9 A 18.15, BVerwGE 156, 215, Rdnr. 83.

41) BVerwG, Urt. v. 8.1.2014 - 9 A 4.13, BVerwGE 149, 31, Rdnr. 99

42) BVerwG, Beschl. v. 8.3.2018 - 9 B 25.17, NuR 2018, 625, Rdnr. 11

43) BVerwG, Urt. v. 9.2.2017 - 7 A 2.15, BVerwGE 158, 1, Rdnr. 466

44) BVerwG, Urt. v. 14.3.2018 - 4 A 5.17, BVerwGE 161, 263 , Rdnr. 70; BVerwG, Urt. v. 6.4.2017 - 4 A 16.16, NuR 2018, 255, Rdnr. 58.

45) BVerwG, Beschl. v. 23.1.2015 - 7 VR 6.14, NuR 2015, 257, Rdnr. 30; BVerwG, Urt. v. 6.4.2017 - 4 A 16.16, NuR 2018, 255, Rdnr. 75; BVerwG, Beschl. v. 8.3.2018 - 9 B 25.17, NuR 2018, 625, Rdnr. 11.

46) BVerwG, Urt. v. 6.4.2017 - 4 A 16.16, NuR 2018, 255 , Rdnr. 75; BVerwG, Beschl. v. 8.3.2018 - 9 B 25.17, NuR 2018, 625, Rdnr. 11.

47) Siehe exemplarisch Bayerisches Landesamt für Umwelt, Arbeitshilfe Vogelschutz und Windenergienutzung, 2017, Stand 20.1.2020, abrufbar unter https://www.landschaft-artenschutz. de/wp-content/uploads/2017-06_Vogelschutz-und-WEA_ LfU.pdf.

48) BVerwG, Urt. v. 22.9.2016 - 4 C 2.16, BVerwGE 156, 148, Rdnr. 35.

49) BVerwG, Beschl. v. 8.3.2018 - 9 B 25.17, NuR 2018, 625, Rdnr. 28; BVerwG, Urt. v. 27.11.2018 - 9 A 8.17, BVerwGE 163, 380, Rdnr. 100 .

50) BVerwG, Beschl. v. 8.3.2018 - 9 B 25.17, NuR 2018, 625, Rdnr. 28; BVerwG, Urt. v. 27.11.2018 - 9 A 8.17, BVerwGE 163, 380, Rdnr. 100.

51) Dierschke/Bernotat, Übergeordnete Kriterien zur Bewertung der Mortalität wildlebender Tiere im Rahmen von Projekten und Eingriffen, 3. Fassung, 20.9.2016, Stand 20.1.2020, abrufbar unter http://www.gavia-ecoresearch.de/ref/pdf/Bernotat_ Dierschke_2016.pdf.

52) BVerwG, Beschl. v. 29.10.2014 - 7 VR 4.13, Rdnr. 15; ähnlich BVerwG, Urt. v. 8.1.2014 - 9 A 4/13, BVerwGE 149, 31 Rdnr. 93.

53) BVerwG, Beschl. v. 29.10.2014 - 7 VR 4.13, Rdnr. 15.

54) BVerwG, Urt. v. 23.4.2014 - 9 A 25.12, BVerwGE 149, 289, Rdnr. 26.

55) BVerwG, Urt. v. 28.4.2016 - 9 A 9.15, BVerwGE 155, 91 Rdnr. 141; BVerwG, Urt. v. 10.11.2016 - 9 A 18.15, BVerwGE 156, 215, Rdnr. 82; BVerwG, Urt. v. 6. 4.2017 - 4 A 16.16, NuR 2018, 255 Rdnr. 77. 
eine Vielzahl an Kriterien in Betracht, die gerichtlich nur auszugsweise abstrakt bestimmt wurden. Dazu fehlen häufig fachlich anerkannte Standards und Erkenntnisse über die Verhaltensweisen der verschiedenen besonders geschützten Arten, insbesondere in Bezug auf neue Anlagen, die den natürlichen Lebensraum von Individuen der besonders geschützten Arten beeinflussen ${ }^{56}$. Auch mangelt es überwiegend an anerkannten einheitlichen Methoden der Bestandserfassung im geplanten Vorhabengebiet. All das führt bei den zuständigen Behörden zu großen Schwierigkeiten in der praktischen Anwendung des artenschutzrechtlichen Tötungsverbots und der rechtlichen Ausfüllung des Signifikanzkriteriums.

Um den Behörden die Möglichkeit zu geben, den Tatbestand des artenschutzrechtlichen Tötungsverbots und dabei das Signifikanzkriterium im Einzelfall anwenden zu können, hat das BVerwG den Behörden bislang zur fachlichen Bewertung des Tötungsverbots eine naturschutzfachliche Einschätzungsprärogative zugestanden ${ }^{57}$. Die Einschätzungsprärogative umfasste im Kern die fachliche Beurteilung, ob das Tötungsrisiko für Individuen besonders geschützter Arten durch das geplante Vorhaben signifikant erhöht war ${ }^{58}$.

\subsubsection{Korrektur der Rechtsentwicklung durch \\ das Bundesverfassungsgericht: Eingeschränkte gerichtliche Kontrolle, aber kein Beurteilungsspielraum}

Diese bisherige Linie der Verwaltungsgerichte hat nunmehr durch das BVerfG eine Korrektur erfahren. In seinem Beschluss vom 23.10.2018 bestätigt das Gericht zwar, dass die eingeschränkte Reichweite der verwaltungsgerichtlichen Kontrolle bezüglich des artenschutzrechtlichen Tötungsverbots in $\$ 44$ Abs. 1 Nr. 1 BNatSchG mit der verfassungsrechtlich in Art. 19 Abs. 4 S. 1 GG verankerten Rechtsschutzgarantie vereinbar ist, jedenfalls ,,wenn die Anwendung eines Gesetzes tatsächliche Feststellungen verlangt, zu denen weder eine untergesetzliche Normierung erfolgt ist, noch in Fachkreisen und Wissenschaft allgemein anerkannte Maßstäbe und Methoden existieren"59. Dennoch besteht kein Beurteilungsspielraum der Behörden.

Zur Absicherung seiner Feststellung argumentiert das BVerfG grundlegend. Es setzt zunächst bei der eingeschränkten gerichtlichen Kontrolle an und zeigt auf, dass Gerichte grundsätzlich verpflichtet sind, beim Fehlen untergesetzlicher Konkretisierungen der gesetzlichen Tatbestandsmerkmale des artenschutzrechtlichen Tötungsverbots sich ,,zur fachlichen Aufklärung der Tatbestandsmerkmale unmittelbar der Erkenntnisse der Fachwissenschaft und -praxis zu bedienen ${ }^{60}$. Kann aber die Fachwissenschaft nicht die erforderlichen Erkenntnisse liefern, müssen und dürfen die Gerichte diese Erkenntnisgrenzen respektieren; denn hier stößt das Gericht „,an objektive Grenzen“. ${ }^{61}$ Aus diesem Befund leitet das BVerfG zweierlei ab: eine Entlastung des Gerichts und eine judikative Zurückhaltung. Die Entlastung betrifft die Sachaufklärungspflicht des entscheidungsberufenen $\mathrm{Ge}-$ richts: „Wenn die gerichtliche Kontrolle nach weitestmöglicher Aufklärung an die Grenze des Erkenntnisstandes der ökologischen Wissenschaft und Praxis stößt, zwingt Art. 19 Abs. 4 S. 1 GG das Gericht nicht zu weiteren Ermittlungen, sondern erlaubt es dem Gericht, seiner Entscheidung insoweit die Einschätzung der Behörde zu der fachlichen Frage zugrunde zu legen, wenn diese auch aus gerichtlicher Sicht plausibel ist" "62. Denn Gerichte sind nicht in der Lage, ,fachwissenschaftliche Erkenntnislücken selbstständig zu schließen, und auch nicht verpflichtet, über Ermittlungen im Rahmen des Stands der Wissenschaft hinaus Forschungsaufträge zu erteilen" 63 . Ob sich Maßstäbe und Methoden im Einzelfall durchgesetzt haben bzw. fehlen, ist jedoch Gegenstand vollständiger gerichtlicher Überprüfung ${ }^{64}$.

Kehrseite einer möglichen Entlastung ist die Rücknahme der gerichtlichen Kontrollbefugnis; insofern muss sich das Gericht mit eigenen Einschätzungen zu ungeklärten wissenschaftlichen Fachfragen zurückhalten, denn es ,bliebe angesichts der unzureichenden (naturschutzfachlichen) Er- kenntnislage zwangsläufig immer noch ungeklärt und auch objektiv unaufklärbar, ob die behördliche Einschätzung oder die gerichtliche Einschätzung richtig ist" 65 . Hinzu kommt, dass in außerrechtlichen, tatsächlichen Fragen anders als in rechtlichen Fragen - ,zugunsten der Gerichtsbarkeit keine Vermutung besteht, dass sie über mehr Expertise verfügt als die Verwaltung"

Trotz aller gerichtlicher Zurückhaltung ruft das BVerfG gleichwohl nicht zur Untätigkeit auf. Die zuständigen Gerichte müssen weiterhin untersuchen, ob „die von der Behörde verwendeten fachlichen Maßstäbe und Methoden vertretbar sind und die Behörde insofern im Ergebnis zu einer plausiblen Einschätzung der fachlichen Tatbestands-

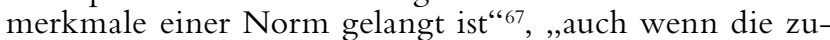
grundeliegende gesetzliche Regelung außerrechtliche fachliche Beurteilungen erfordert" ${ }^{68}$ Darüber hinaus bleiben die Gerichte verpflichtet zu überprüfen, ob „,der Behörde bei der Ermittlung und der Anwendung der von ihr aus dem Spektrum des Vertretbaren gewählten fachlichen Methode Verfahrensfehler unterlaufen, ob sie anzuwendendes Recht verkennt, von einem im Übrigen unrichtigen oder nicht hinreichend tiefgehend aufgeklärten Sachverhalt ausgeht, allgemeingültige Bewertungsmaßstäbe verletzt oder sich von sachfremden Erwägungen leiten lässt" 69 .

Als dogmatische Begründung für die dargestellte Rücknahme der gerichtlichen Kontrolle greift das BVerfG nicht auf die vom BVerwG geprägte Rechtsfigur der natur-

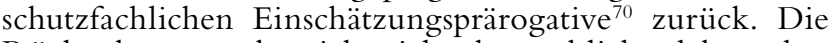
Rücknahme ergebe sich vielmehr ,,schlicht daher, dass sich die naturschutzfachliche Richtigkeit des Ergebnisses der Verwaltungsentscheidung objektiv nicht abschlieBend beurteilen lässt" ${ }^{\text {"11 }}$. Bei der eingeschränkten gerichtlichen Kontrolle von Behördenentscheidungen im Rahmen des artenschutzrechtlichen Tötungsverbots handelt es sich also gerade nicht um eine - für die Annahme eines Beur-

56) BVerfG, Beschl. v. 23.10.2018 - 1 BvR 2523/13 und 1 BvR 595/14, BVerfGE 149, 407, Rdnr. $31 \mathrm{ff}$.

57) BVerwG, Urt. v. 28.4.2016 - 9 A 9.15, BVerwGE 155, 91, Rdnr. 141; BVerwG, Urt. v. 10.11.2016 - 9 A 18.15, BVerwGE 156, 215, Rdnr. 84; BVerwG, Urt. v. 6. 4.2017 - 4 A 16.16, NuR 2018, 255, Rdnr. 74

58) BVerwG, Urt. v. 28.4.2016 - 9 A 9.15, BVerwGE 155, 91, Rdnr. 141; BVerwG, Urt. v. 10.11.2016 - 9 A 18.15, BVerwGE 156, 215, Rdnr. 84.

59) BVerfG, Beschl. v. 23.10.2018 - $1 \mathrm{BvR} 2523 / 13$ und $1 \mathrm{BvR}$ 595/14, BVerfGE 149, 407, Rdnr. 16.

60) BVerfG, Beschl. v. 23.10.2018 - 1 BvR 2523/13 und 1 BvR 595/14, BVerfGE 149, 407, Rdnr. 27.

61) BVerfG, Beschl. v. 23.10.2018 - 1 BvR 2523/13 und $1 \mathrm{BvR}$ 595/14, BVerfGE 149, 407, Rdnr. 20.

62) BVerfG, Beschl. v. 23.10.2018 - 1 BvR 2523/13 und 1 BvR 595/14, BVerfGE 149, 407, Rdnr. 18.

63) BVerfG, Beschl. v. 23.10.2018 - $1 \mathrm{BvR} 2523 / 13$ und $1 \mathrm{BvR}$ 595/14, BVerfGE 149, 407, Rdnr. 20.

64) BVerfG, Beschl. v. 23.10.2018 - 1 BvR 2523/13 und 1 BvR 595/14, BVerfGE 149, 407, Rdnr. 27 = NVwZ 2019, 52, $54 \mathrm{f}$.

65) BVerfG, Beschl. v. 23.10.2018 - 1 BvR 2523/13 und 1 BvR 595/14, BVerfGE 149, 407, Rdnr. 22

66) BVerfG, Beschl. v. 23.10.2018 - 1 BvR 2523/13 und 1 BvR 595/14, BVerfGE 149, 407, Rdnr. 22.

67) BVerfG, Beschl. v. 23.10.2018 - 1 BvR 2523/13 und 1 BvR 595/14, BVerfGE 149, 407, Rdnr. 28. So bereits BVerwG, Urt. v. 28.4.2016 - 9 A 9.15, BVerwGE 155, 91, Rdnr. 128.

68) BVerfG, Beschl. v. 23.10.2018 - 1 BvR 2523/13 und 1 BvR 595/14, BVerfGE 149, 407, Rdnr. 19.

69) BVerfG, Beschl. v. 23.10.2018 - 1 BvR 2523/13 und 1 BvR 595/14, BVerfGE 149, 407, Rdnr. 30.

70) BVerwG, Urt. v. 28.4.2016 - 9 A 9.15, BVerwGE 155, 91, Rdnr. 141; BVerwG, Urt. v. 10.11.2016 - 9 A 18.15, BVerwGE 156, 215, Rdnr. 84; BVerwG, Urt. v. 6. 4. 2017 - 4 A 16.16, NuR 2018, 255, Rdnr. 74

71) BVerfG, Beschl. v. 23.10.2018 - 1 BvR 2523/13 und 1 BvR 595/14, BVerfGE 149, 407, Rdnr. 17. 
teilungsspielraums erforderliche - ,gewillkürte Verschiebung der Entscheidungszuständigkeit vom Gericht auf die Behörde, sondern um eine nach Dauer und Umfang vom jeweiligen ökologischen Erkenntnisstand abhängige faktische Grenze verwaltungsgerichtlicher Kontrolle“72.

Überträgt man diese Überlegungen auf das artenschutzrechtliche Tötungsverbot, führt dies zu einer Begrenzung der gerichtlichen Kontrolle, da sich die Beurteilung des Tötungsrisikos nach außerrechtlichen Kriterien richtet, die bislang aber noch in weiten Teilen fehlen ${ }^{73}$.

\subsubsection{Einzelfragen zur eingeschränkten gerichtlichen Kontrolle}

Welche Konturen sich für die eingeschränkte gerichtliche Kontrolle nunmehr ergeben, wurde zwar prinzipiell durch das BVerfG dargestellt. Einzelfragen bleiben aber offen. Es liegt nahe, die bisherigen Ausführungen des BVerwG zur Einschätzungsprärogative zur Orientierung heranzuziehen.

Somit dürfte es auch weiterhin den Behörden möglich sein, ergänzend Kriterien zu definieren, die ,in Zusammenhang mit der Biologie der Art stehen"74, wenn es um die Bestimmung einer ,signifikanten Erhöhung des Tötungsrisikos" im Sinne des $\$ 44$ Abs. 5 S. 2 Nr. 1 BNatSchG geht. Gleiches dürfte für die naturschutzfachliche Einschätzung gelten, ob und welche Wirkung etwaige Schadensvermeidungsmaßnahmen haben ${ }^{75}$. Dies ist konsequent, da die Wirkung von Schadensvermeidungsmaßnahmen in die Beurteilung, ob das Tötungsrisiko durch das geplante Vorhaben signifikant erhöht ist, mit einzubeziehen ist. ${ }^{76}$

Weitere gerichtliche Zurückhaltung ist gefordert, wenn es um die Frage geht, wie der Bestand der besonders geschützten Arten im Gebiet des geplanten Vorhabens erfasst wird und wie die Risiken und Betroffenheiten für die möglicherweise geschädigten Individuen bewertet wer$\operatorname{den}^{77}$. Gleiches gilt, wenn die Behörde keine vollständige Untersuchung des Vorhabenraums vornimmt, sondern bereits gesammelte Erkenntnisse und durchgeführte Untersuchungen des betroffenen Vorhabengebiets aktualisiert ${ }^{78}$.

Fachliche Unsicherheiten bzw. Zweifelslagen entbinden die Exekutive nicht davon unter Beachtung der geltenden wissenschaftlichen Standards ,,alle wissenschaftlichen Mittel und Quellen“ auszuschöpfen ${ }^{79}$; gewählte Methoden müssen fachwissenschaftlich vertretbar $\operatorname{sein}^{80}$, wobei „eine naturschutzfachliche Methodik einer anderen vertretbaren Einschätzung nicht schon deshalb vorzugswürdig ist, weil sie umfangreichere oder aufwändigere Ermittlungen und

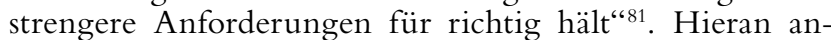
knüpfend müssen die Gerichte überprüfen, ob - im Fall mangelnder fachwissenschaftlicher Aufarbeitung der Sachfragen - die ,konkreten Untersuchungen der Behörde in ihrer Ermittlungstiefe und ihrem methodischen Vorgehen dazu geeignet sind, die Behörde in die Lage zu versetzen, die Voraussetzungen der artenschutzrechtlichen Verbotstatbestände sachgerecht zu überprüfen" $" 82$.

\subsubsection{Verpflichtung des Gesetzgebers}

Die Steuerungslücken, die durch fehlende Erkenntnisse zu den naturfachlichen Gegebenheiten entstehen und im Einzelfall von der Exekutive zu füllen sind, erschweren nicht nur die praktische Arbeit und erzeugen Unsicherheit auf Seiten von Vorhabenträgern. Sie werfen mit Blick auf die kaum präzisierten Tatbestandsmerkmale auch verfassungsrechtliche Probleme auf, denn ,ein Gesetz, welches solche auf ungeklärte naturschutzfachliche Zusammenhänge verweisende Tatbestandsmerkmale verwendet, (wirft) mit Blick auf die materiellen Grundrechte und den aus Demokratie- und Rechtsstaatsprinzip abgeleiteten Wesentlichkeitsgrundsatz (...) verfassungsrechtliche Zweifel auf" 83 .

Deshalb nimmt das BVerfG die Legislative in die Pflicht: Der Gesetzgeber darf ,,in grundrechtsrelevanten Bereichen (...) der Rechtsanwendung nicht ohne weitere Maßgaben auf Dauer Entscheidungen in einem fachwissenschaftlichem ,Erkenntnisvakuum' übertragen, das weder Verwaltung noch
Gerichte selbst auszufüllen vermögen" ${ }^{44}$. Und das BVerfG wird noch präziser: „Der Gesetzgeber mag, je nach Grundrechtsbetroffenheit, kurzfristig darauf vertrauen können, dass sich fachliche Wissenslücken durch Erkenntnisfortschritte in Fachkreisen und Wissenschaft schließen. Längerfristig dürfte der Gesetzgeber dem jedoch nicht tatenlos zusehen, weil er sich so seiner inhaltlichen Entscheidungsverantwortung entzieht, privatem Fachwissen ungesteuert weitreichenden Einfluss auf staatliche Entscheidungen eröffnet und eine einheitliche Rechtsanwendung nicht gewährleistet ist. Der Gesetzgeber muss dann, sofern die fachlichen Zusammenhänge weiter ungeklärt sind, für eine zumindest untergesetzliche Maßstabsbildung beispielsweise durch Einsetzung fachkundiger Gremien zur Festlegung einheitlicher Maßstäbe und Methoden sorgen oder wenigstens genauere Regeln für die behördliche Entscheidung zwischen mehreren vertretbaren Auffassungen vorgeben" ${ }^{85}$. Leider erklärt das BVerfG nicht die rechtlichen Konsequenzen, die sich ergeben, wenn der Gesetzgeber trotz dieses eindeutigen Auftrags keine Maßstäbe schafft. Der Frage nachzuspüren, würde den vorliegenden Rahmen sicher sprengen. Soviel nur: Da ein Regelungsverzicht sicher keinen Genehmigungsanspruch auf Seiten der Grundrechtsbetroffenen bewirkt, werden die durchgehend ablehnenden Haltungen zu einer gegen die Legislative gerichtete Normerlassklage oder zu einem gegen sie gerichteten Haftungsanspruch zumindest unter Druck geraten. ${ }^{86}$

72) BVerfG, Beschl. v. 23.10.2018 - 1 BvR 2523/13 und 1 BvR 595/14, BVerfGE 149, 407, Rdnr. 23.

73) BVerfG, Beschl. v. 23.10.2018 - 1 BvR 2523/13 und $1 \mathrm{BvR}$ 595/14, BVerfGE 149, 407, Rdnr. 32.

74) Noch unter der Prämisse einer behördlichen Einschätzungsprärogative: BVerwG, Urt. v. 6. 4.2017 - 4 A 16.16, NuR 2018, 255, Rdnr. 75; BVerwG, Beschl. v. 8.3.2018 - 9 B 25.17, NuR 2018, 625, Rdnr. 11

75) Noch unter der Prämisse einer behördlichen Einschätzungsprärogative: BVerwG, Beschl. v. 8.3.2018 - 9 B 25.17, NuR 2018, 625, Rdnr. 17.

76) Noch unter der Prämisse einer behördlichen Einschätzungsprärogative: BVerwG, Beschl. v. 23.1.2015 - 7 VR 6.14, NuR 2015 , 257, Rdnr. 30; BVerwG, Urt. v. 6.4.2017 - 4 A 16.16, NuR 2018, 255, Rdnr. 75; BVerwG, Beschl. v. 8.3.2018 - 9 B 25.17, NuR 2018, 625, Rdnr. 17

77) Noch unter der Prämisse einer behördlichen Einschätzungsprärogative: BVerwG, Urt. v. 10.11.2016 - 9 A 18.15, BVerwGE 156, 215, Rdnr. 73.

78) Noch unter der Prämisse einer behördlichen Einschätzungsprärogative: BVerwG, Urt. v. 25.6.2014 - 9 A 1.13, BVerwGE 150, 92, Rdnr. 37.

79) Noch unter der Prämisse einer behördlichen Einschätzungsprärogative: BVerwG, Urt. v. 23. 4. 2014 - 9 A 25.12, BVerwGE 149, 289, Rdnr. 26.

80) Noch unter der Prämisse einer behördlichen Einschätzungsprärogative: BVerwG, Beschl. v. 8.3.2018 - 9 B 25.17, NuR 2018, 625, Rdnr. 25

81) Noch unter der Prämisse einer behördlichen Einschätzungsprärogative: BVerwG, Urt. v. 28.4.2016 - 9 A 9.15, BVerwGE 155, 91, Rdnr. 134; BVerwG, Urt. v. 10.11.2016 - 9 A 18.15, BVerwGE 156, 215, Rdnr. 76

82) Noch unter der Prämisse einer behördlichen Einschätzungsprärogative: BVerwG, Beschl. v. 8.3.2018 - 9 B 25.17, NuR 2018, 625, Rdnr. 25.

83) BVerfG, Beschl. v. 23.10.2018 - 1 BvR 2523/13 und 1 BvR 595/14, BVerfGE 149, 407, Rdnr. 24.

84) BVerfG, Beschl. v. 23.10.2018 - 1 BvR 2523/13 und 1 BvR 595/14, BVerfGE 149, 407, Rdnr. 24.

85) BVerfG, Beschl. v. 23.10.2018 - 1 BvR 2523/13 und 1 BvR 595/14, BVerfGE 149, 407, Rdnr. 24.

86) $\mathrm{Zu}$ legislativem Unrecht bei Amtshaftungsansprüchen siehe $\mathrm{Os}$ senbühl/Cornils, Staatshaftungsrecht, 2013, S. 106f; zu legislativem Unrecht bei enteignungsgleichem Eingriff ebenda S. 281 f; zur Entschlussfreiheit der Legislative hinsichtlich des Erlasses eines formellen Gesetzes siehe Ahrens, Staatshaftungsrecht, 2018 , Rdnr. 58 sowie Papier/Shirvani, in: Münchener Kommentar zum BGB, 2017, \$839 Rdnr. 261. 
4.2 Vermeidungsmaßnahmen,

S44 Abs. 5 S. 2 Nr. 1 BNatSch G

Ein Verstoß gegen das artenschutzrechtliche Tötungsverbot aus $₫ 44$ Abs. 1 Nr. 1 BNatSchG liegt nach $\ 44$ Abs. 5 S. 2 Nr. 1 BNatSchG nicht vor, wenn die signifikante Erhöhung des Tötungsrisikos bei Anwendung der gebotenen, fachlich anerkannten Schutzmaßnahmen vermieden werden kann. Aus diesem Grund muss die Wirksamkeit vorgesehener Vermeidungsmaßnahmen als besonderer Umstand in die Gesamtbetrachtung, ob das Tötungsrisiko im Einzelfall signifikant erhöht ist, eingestellt werden. Konkret wird untersucht, ob Tötungen durch die vorgesehenen Maßnahmen derart umfänglich vermieden werden können, dass die prognostizierten Tötungen innerhalb eines Risikobereichs verbleiben, die dem allgemeinen Risiko für Individuen entsprechen ${ }^{87}$. Hierbei ist nach Ansicht des BVerwG nicht zu fordern, dass die vorgesehenen Schutzmaßnahmen die prognostizierten Tötungen mit 100prozentiger Wahrscheinlichkeit vermeiden müssen. ${ }^{8}$ Diese Sichtweise ist stimmig, da auch die Ablehnung des Tatbestandsmerkmals der Signifikanz gerade kein Nullrisiko erfordert ${ }^{89}$. Vermeidungsmaßnahmen müssen aber in jedem Fall dem Verhältnismäßigkeitsgrundsatz entsprechen $^{90}$. Dies führt in eine Einzelfallbetrachtung. So wurde etwa die Wirksamkeit von Überflughilfen, Kollisionsschutzwänden, Wildschutzzäunen und Leiteinrichtungen als Schadensvermeidungsmaßnahmen im Sinne von $₫ 44$ Abs. 5 S. 2 Nr. 1 BNatSchG bei der geplanten Realisierung von Bundesautobahnen im Außenbereich ausdrücklich anerkannt ${ }^{91}$ und damit die Möglichkeit der tatbestandsausschließenden Wirkung für diese konkreten Vermeidungsmaßnahmen eröffnet.

\subsection{Das Störungsverbot nach $\int 44$ Abs. 1 Nr. 2 BNatSchG} 4.3.1 Allgemeines

Auch das artenschutzrechtliche Störungsverbot gemäß \$44 Abs. 1 Nr. 2 BNatSchG war in den letzten Jahren Gegenstand der höchstrichterlichen Jurisdiktion. Das artenschutzrechtliche Störungsverbot ist - wie das artenschutzrechtliche Tötungsverbot - als ordnungsrechtliche Schutzvorschrift ausgestaltet, die der Genehmigung geplanter Vorhaben entgegenstehen kann. Im Unterschied zum artenschutzrechtlichen Tötungsverbot sind von der Schutzwirkung des artenschutzrechtlichen Störungsverbots gem. $\$ 44$ Abs. 1 Nr. 2 BNatSchG nur wildlebende Tiere der streng geschützten Arten und der europäischen Vogelarten $^{92}$ umfasst, was einerseits tatbestandlich einschränkend wirkt, andererseits aber eine strenge Schutzwirkung auslöst. Die strenge Schutzwirkung des $\ 44$ Abs. 1 Nr. 2 BNatSchG kann so weit reichen, dass bei selten vorkommenden Arten bereits ein einzelnes Brutpaar eine im Einzelfall beachtenswerte lokale Population im Sinne von $\$ 44$ Abs. 1 Nr. 2 HS 2 BNatSchG darstellen kann ${ }^{93}$.

Allerdings sieht der Tatbestand des artenschutzrechtlichen Störungsverbots ein die Schutzwirkung einschränkendes zeitliches Element vor, da die wildlebenden Tiere nur in den in $\$ 44$ Abs. 1 Nr. 2 BNatSchG vorgesehenen Zeitperioden, wie beispielsweise der Fortpflanzungszeit oder der Überwinterungszeit, vor Störungen zu schützen sind. Über die Interpretation dieser Zeitperioden besteht weiterhin Unsicherheit. Das BVerwG hat sein Verständnis bislang nur angedeutet: Zum Begriff der „Fortpflanzungszeit" legt es etwa dar, dass die geschützte Fortpflanzungszeit im Einzelfall dann betroffen sein könnte, ,wenn dieser Begriff dahin verstanden wird, dass er den Zeitraum vom Beginn des Werbeverhaltens bis zum Abschluss des Brutgeschäfts - sei es erfolgreich oder erfolglos - oder bis zum Ende der Trächtigkeit umfasst"94 und vorgesehene Maßnahmen, die eine Störung darstellen könnten, ,nach Beginn des Werbeverhaltens erfolgen sollten" ${ }^{\text {"95. }}$. Letztlich hat der 9. Senat die Frage aber mangels Entscheidungserheblichkeit offengelassen.
Dem Wortlaut des $₫ 44$ Abs. 1 Nr. 2 HS 2 BNatSchG ist $\mathrm{zu}$ entnehmen, dass der Tatbestand des artenschutzrechtlichen Störungsverbots nur dann erfüllt ist, wenn sich der Erhaltungszustand der lokalen Population einer Art verschlechtert. Demnach verfolgt das artenschutzrechtliche Störungsverbot einen populationsbezogenen Ansatz ${ }^{96}$ und unterscheidet sich insoweit vom artenschutzrechtlichen Tötungsverbot nach $₫ 44$ Abs. $1 \mathrm{Nr} .1 \mathrm{BNatSchG}$, das einen individuenbezogenen Ansatz wählt. Diese Differenzierung ist aus Sicht des BVerwG unionsrechtlich unbedenklich ${ }^{97}$, da auch die europäischen Richtlinien einen ,,art- bzw. populationsbezogenen Schutzansatz verfolgen“" und ,diese Auffassung von der Europäischen Kommission geteilt" wird ${ }^{98}$.

\subsubsection{Einzelfragen}

Eine ,erhebliche Störung“ im Sinne von $₫ 44$ Abs. 1 Nr. 2 BNatSchG kann ,vor allem durch bau- und betriebsbedingte Beeinträchtigungen der geschützten Tierarten in Gestalt von akustischen und optischen Störwirkungen" "99 eintreten. Tatbestandlich können laut BVerwG ferner Trennwirkungen innerhalb des natürlichen Lebensraums von Tieren der besonders geschützten Arten sein, die durch die Umsetzung des geplanten Vorhabens entstehen würden ${ }^{100}$. Selbst Maßnahmen, die angeordnet worden sind, um die Erfüllung der Tatbestandsvoraussetzungen des artenschutzrechtlichen Tötungsverbots ( $\$ 44$ Abs. $1 \mathrm{Nr} .1 \mathrm{BNatSchG})$ zu vermeiden, können gegen das Störungsverbot des $₫ 44$ Abs. 1 Nr. 2 BNatSchG verstoßen ${ }^{101}$. Der Anordnung einer solchen Vermeidungsmaßnahme im Sinne von $₫ 44$ Abs. 5 S. 2 Nr. 1 BNatSchG haftet keine Unbedenklichkeitsbescheinigung hinsichtlich einer Nichterfüllung anderer artenschutzrechtlicher Verbote an. Nach Ansicht des BVerwG spielt nämlich die Motivation der Vermeidungsmaßnahme keine Rolle für ihre rechtliche Beurteilung; weder dem Wortlaut der betreffenden Normen noch den europäischen Vorlagen (Art. 12 Abs. 1 lit. b) FFH-Richtlinie und Art. 5 lit. b) EG-Vogelschutzrichtlinie) ist eine andere Auslegung des Zusammenspiels von $₫ 44$ Abs. 5 S. 2 Nr. 1 BNatSchG und $\ 44$ Abs. 1 Nr. 2 BNatSchG zu entnehmen ${ }^{102}$.

87) BVerwG, Beschl. v. 8.3.2018 - 9 B 25.17, NuR 2018, 625, Rdnr. 11; BVerwG, Urt. v. 6.4.2017 - 4 A 16.16, NuR 2018, 255, Rdnr. 73, 75.

88) BVerwG, Urt. v. 28.4.2016 - 9 A 9.15, BVerwGE 155, 91 , Rdnr. 141.

89) BVerwG, Urt. v. 28.4.2016 - 9 A 9.15, BVerwGE 155, 91 , Rdnr. 141.

90) BVerwG, Beschl. v. 29.10. 2014 - 7 VR 4.13, Rdnr. 26.

91) BVerwG, Urt. v. 28.4.2016 - 9 A 9.15, BVerwGE 155, 91, Rdnr. 144; BVerwG, Urt. v. 23. 4.2014 - 9 A 25.12, NuR 2014, 706, Rdnr. 102

92) Zur Definition siehe $₫ 7$ Abs. 2 Nr. 14 BNatSchG

93) BVerwG, Urt. v. 27.11.2018 - 9 A 8.17, BVerwGE 163, 380, Rdnr. 127.

94) BVerwG, Urt. v. 27.11.2018 - 9 A 8.17, BVerwGE 163, 380, Rdnr. 126.

95) BVerwG, Urt. v. 27.11.2018 - 9 A 8.17, BVerwGE 163, 380, Rdnr. 126.

96) BVerwG, Urt. v. 6.4.2017 - 4 A 16.16, NuR 2018, 255, Rdnr. 80; BVerwG, Urt. v. 9.2.2017 - 7 A 2.15, BVerwGE 158, 1, Rdnr. 471.

97) BVerwG, Urt. v. 6.4.2017 - 4 A 16.16, NuR 2018, 255, Rdnr. 80.

98) BVerwG, Urt. v. 6.4.2017 - 4 A 16.16, NuR 2018, 255, Rdnr. 80.

99) BVerwG, Urt. v. 6.4.2017 - 4 A 16.16, NuR 2018, 255 , Rdnr. 80; BVerwG, Urt. v. 9.2.2017 - 7 A 2.15, BVerwGE 158, 1, Rdnr. 471.

100) BVerwG, Urt. v. 6.4.2017 - 4 A 16.16, NuR 2018, 255, Rdnr. 80.

101) BVerwG, Urt. v. 27.11.2018 - 9 A 8.17, BVerwGE 163, 380, Rdnr. 125.

102) BVerwG, Urt. v. 27.11.2018 - 9 A 8.17, BVerwGE 163, 380, Rdnr. 125. 
Bei der Bewertung der Frage, ob eine Störung von wildlebenden Tieren streng geschützter Arten vorliegt, die den Erhaltungszustand der lokalen Population einer Art verschlechtert, dürfte zwar keine Einschätzungsprärogative anzunehmen, ${ }^{103}$ wohl aber die Kontrollbefugnis der Gerichte zurückgenommen sein. ${ }^{104}$ Grund hierfür ist, dass auch hinsichtlich der Bewertung der Fragen, die zur Tatbestandserfüllung des artenschutzrechtlichen Störungsverbots notwendigerweise zu beantworten sind, keine gesicherten naturschutzfachlichen Kenntnisse existieren und man sich auch in diesem Bereich in einem ,Erkenntnisvakuum“ befinde ${ }^{105}$. Derartige Kenntnislücken zur Beantwortung naturschutzfachlicher Fragen können durch Wissenstransfers aus anderen Bereichen geschlossen werden. ,,(D)er Analogieschluss (ist) eine gängige und unbedenkliche Methode, mit der bei Einhaltung wissenschaftlicher Standards (die bestehenden naturschutzfachlichen) Kenntnislücken überbrückt werden können" ${ }^{106}$.

\subsection{Das Zerstörungsverbot nach $\int 44$ Abs. 1 Nr. 3 BNatSchG}

Auch das artenschutzrechtliche Zerstörungsverbot aus $\$ 44$ Abs. 1 Nr. 3 BNatSchG kann als ordnungsrechtliche Schutzvorschrift einem genehmigungsbedürftigen Vorhaben im Einzelfall entgegenstehen, sodass auch diese artenschutzrechtliche Verbotsnorm eine erhöhte praktische Relevanz aufweist. Das artenschutzrechtliche Zerstörungsverbot wird etwa dann verwirklicht, wenn zu Lasten wildlebender Tiere der besonders geschützten Arten ( $\$ 44$ Abs. 1 Nr. 3 BNatSchG) ,durch das geplante Vorhaben in mindestens einem regelmäßig belegten Brutrevier alle als Standort von Nestern geeigneten Brutplätze verloren gingen “107, wobei der Begriff der Ruhe- oder Fortpflanzungsstätte im Sinne von $\$ 44$ Abs. 1 Nr. 3 BNatSchG eng auszulegen ist. ${ }^{108}$ Der Terminus umfasst ,nicht den allgemeinen Lebensraum der geschützten Arten und sämtliche Lebensstätten, sondern einen abgrenzbaren und für die betroffene Art besonders wichtigen Fortpflanzungs- und Ruhebereich" 109 , schließt aber ,,jeden einem Zugriff zugänglichen, als Ort der Fortpflanzung dienenden Gegenstand und damit auch einzelne Nester ein"110. Nahrungs-, Jagd und potenzielle Lebensstätten sowie Wanderkorridore sind nicht von der Schutzwirkung des $₫ 44$ Abs. 1 Nr. 3 BNatSchG umfasst ${ }^{111}$.

Nach Ansicht des BVerwG ist zu fordern, dass ein Fortpflanzungs- und Ruhebereich im Sinne von $\$ 44$ Abs. 1 Nr. 3 BNatSchG, ,einen nicht nur vorübergehenden, den artspezifischen Ansprüchen genügenden störungsfreien Aufenthalt ermöglicht $^{\text {" } 112}$. Ein Verstoß gegen $\ 44$ Abs. 1 Nr. 3 BNatSchG liegt also nicht vor, ,wenn die ökologische Funktion der von dem Vorhaben betroffenen Fortpflanzungs- und Ruhestätten im räumlichen Zusammenhang weiterhin erfüllt wird"113. Soweit erforderlich können deshalb zur Bewahrung oder Wiederherstellung der ökologischen Funktion der von dem Vorhaben betroffenen Fortpflanzungs- und $\mathrm{Ru}-$ hestätten vorgezogene Ausgleichsmaßnahmen (,CEF-Maßnahmen“) festgesetzt werden, um einen Verstoß gegen $₫ 44$ Abs. 1 Nr. 3 BNatSchG zu vermeiden ${ }^{114}$.

Das BVerwG hat sich mit der Schutzwirkung des $\$ 44$ Abs. 1 Nr. 3 BNatSchG auch in zeitlicher Hinsicht befasst. Demnach betrifft $\ 44$ Abs. 1 Nr. 3 BNatSchG, ,primär die Phase aktueller Nutzung der Lebensstätte" "115. Allerdings ist der Schutz ,unter Berücksichtigung des durch $\$ 44$ Abs. 1 Nr. 3 BNatSchG verfolgten Zwecks, die Funktion der Lebensstätte für die geschützte Art zu sichern, auszudehnen auf Abwesenheitszeiten der die Lebensstätte nutzenden Tiere einer Art, sofern nach deren Lebensgewohnheiten eine regelmäßig wiederkehrende Nutzung zu erwarten ist" ${ }^{\text {"116 }}$. Ist eine betroffene Vogelart dafür bekannt, jährlich ihre Neststandorte zu wechseln, ist eine Fortpflanzungsstätte jedenfalls dann im Sinne von $\$ 44$ Abs. 1 Nr. 3 BNatSchG betroffen, ,wenn ein ganzes Brutrevier beseitigt wird, in dem sich regelmäßig genutzte Brutplätze be- finden“"117. Welche Voraussetzungen an die „Regelmäßigkeit" der Nutzung von Ruhe- und Fortpflanzungsstätten im Sinne des $\ 44$ Abs. 1 Nr. 3 BNatSchG zu stellen sind, hat das BVerwG bislang nicht beantwortet.

\section{Entscheidungen zur artenschutzrechtlichen Ausnahme gemäß $₫ 45$ Abs. 7 BNatSchG}

Die artenschutzrechtliche Ausnahme gemäß \45 Abs. 7 BNatSchG erlaubt ausnahmsweise, von den artenschutzrechtlichen Zugriffsverboten aus $\$ 44$ Abs. 1 BNatSchG zu befreien. Aufgrund der Ungewissheit hinsichtlich der naturschutzfachlichen Bewertung der Kriterien, die zur Bestimmung der Tatbestandsmerkmale der artenschutzrechtlichen Zugriffsverbote relevant sind, greifen immer mehr Behörden auf die Möglichkeit einer Ausnahmeerteilung zurück, um sich der lästigen Abgrenzungsfragen zu entledigen. In der Praxis gewinnt die artenschutzrechtliche Ausnahme daher zunehmend an Gewicht.

Entgegen dieser Tendenz betont das BVerwG, dass eine artenschutzrechtliche Ausnahme nach $₫ 45$ Abs. 7 BNatSchG nicht erforderlich (und damit unzulässig) ist, wenn die Erfüllung des artenschutzrechtlichen Tötungsverbots durch Schutzmaßnahmen im Sinne von $₫ 44$ Abs. 5 S. 2 Nr. 1 HS 2 BNatSchG vermieden wird ${ }^{118}$. Dies ist konsequent, da ein Ausnahmeverfahren nach $₫ 45$ Abs. 7 BNatSchG die Tatbestandserfüllung eines Zugriffsverbots voraussetzt, die Vornahme wirksamer Schutzmaßnahmen im Sinne von $₫ 44$ Abs. 5 S. 2 Nr. 1 HS 2 BNatSchG dessen Wortlaut nach aber eine Tatbestandserfüllung ausschließt.

Von besonderer Bedeutung in der höchstrichterlichen Rechtsprechung waren außerdem die Anforderungen an die Zumutbarkeit von Alternativstandorten für das Vorhaben im Sinne von $₫ 45$ Abs. 7 S. 2 BNatSchG. Nach $₫ 45$ Abs. 7 S. 2 BNatSchG darf eine artenschutzrechtliche Ausnahme nur zugelassen werden, wenn zumutbare Alternativen nicht ge-

103) So noch BVerwG, Urt. v. 9.11.2017 - 3 A 4.15, BVerwGE 160, 263, Rdnr. 56.

104) Siehe hierzu die obigen Ausführungen unter IV.1.b.

105) Vgl. BVerwG, Urt. v. 27.11.2018 - 9 A 8.17, BVerwGE 163, 380, Rdnr. 133.

106) BVerwG, Urt. v. 27.11.2018 - 9 A 8.17, BVerwGE 163, 380, Rdnr. 133.

107) BVerwG, Urt. v. 25.6.2014 - 9 A 1.13, BVerwGE 150, 92, Rdnr. 41.

108) BVerwG, Urt. v. 9.2.2017 - 7 A 2.15, BVerwGE 158, 1 , Rdnr. 475; BVerwG, Urt. v. 28.4.2016 - 9 A 9.15, BVerwGE 155, 91, Rdnr. 151; BVerwG Urt. v. 6. 4.2017 - 4 A 16.16, NuR 2018, 255, Rdnr. 82.

109) BVerwG, Urt. v. 9.2.2017 - 7 A 2.15, BVerwGE 158, 1, Rdnr. 475; BVerwG, Urt. v. 28.4.2016 - 9 A 9.15, BVerwGE 155, 91, Rdnr. 151.

110) BVerwG, Urt. v. 9.11.2017 - 3 A 4.15, BVerwGE 160, 263, Rdnr. 50.

111) BVerwG, Urt. v. 9.2.2017 - 7 A 2.15, BVerwGE 158, 1, Rdnr. 475.

112) BVerwG, Urt. v. 9.2.2017 - 7 A 2.15, BVerwGE 158, 1 , Rdnr. 475; BVerwG, Urt. v. 28.4.2016 - 9 A 9.15, BVerwGE 155, 91, Rdnr. 151.

113) BVerwG, Urt. v. 9.11.2017 - 3 A 4.15, BVerwGE 160, 263 , Rdnr. 50.

114) BVerwG, Urt. v. 9.11.2017 - 3 A 4.15, BVerwGE 160, 263 , Rdnr. 50.

115) BVerwG, Urt. v. 9.11.2017 - 3 A 4.15, BVerwGE 160, 263, Rdnr. 50; BVerwG, Urt. v. 6.4.2017 - 4 A 16.16, NuR 2018, 255, Rdnr. 82.

116) BVerwG, Urt. v. 9.11.2017 - 3 A 4.15, BVerwGE 160, 263, Rdnr. 50; BVerwG, Urt. v. 6.4.2017 - 4 A 16.16, NuR 2018, 255, Rdnr. 82.

117) BVerwG, Urt. v. 9.11.2017 - 3 A 4.15, BVerwGE 160, 263, Rdnr. 50.

118) BVerwG, Urt. v. 23.4.2014 - 9 A 25.12, BVerwGE 149, 289, Rdnr. 102. 
geben sind. Der Begriff der „zumutbaren Alternative“ bezieht sich auf einen alternativen Standort für das geplante Vorhaben ${ }^{119}$, dessen etwaiges Vorliegen im Ausnahmeverfahren evaluiert werden muss. Auf eine Alternativlösung muss sich der Vorhabenträger jedoch nicht verweisen lassen, „wenn sich die artenschutzrechtlichen Schutzvorschriften am Alternativstandort als ebenso wirksame Zulassungssperre erweisen wie an dem von ihm [ursprünglich] gewählten Stand-

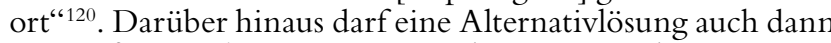
verworfen werden, ,wenn sie sich aus naturschutzexternen Gründen als unverhältnismäßiges Mittel erweist" ${ }^{\text {"121 }}$. Der Verhältnismäßigkeitsgrundsatz ist also weit zu verstehen, sodass auch andere öffentlich-rechtliche Gesichtspunkte in die Frage der Zumutbarkeit eines Alternativstandorts im Sinne von $\ 45$ Abs. 7 S. 2 BNatSchG einfließen müssen.

Das BVerwG hat sich des Weiteren mit $₫ 45$ Abs. 7 S. 3 BNatSchG näher auseinandergesetzt. Das Gericht erläutert, dass Art. 9 Abs. 2 Vogelschutzrichtlinie durch die Verweisung in $\int 45$ Abs. 7 Satz 3 BNatSchG, ,zu einer unmittelbar anwendbaren Norm des nationalen Rechts" wird ${ }^{122}$ und dass ,der Sache des Unionsrechts (...) durch die Verweisung auf Art. 9 Abs. 2 Vogelschutzrichtlinie nicht weniger gedient (ist), als sie es wäre, wenn der Wortlaut des Art. 9 Abs. 2 Vogelschutzrichtlinie in einer Vorschrift des nationalen Rechts wiederholt würde" ${ }^{123}$. Diesem Umstand darf man entnehmen, dass die Verweisung in $\$ 45$ Abs. 7 S. 3 BNatSchG ausreicht, um den Anforderungen des Unionsrechts gerecht zu werden.

\section{Entscheidungen zu Befreiung ( $\$ 67 \mathrm{BNatSchG)}$ und Entschädigung ( $\$ 68$ Abs. 1 BNatSchG)}

Abschließend soll der Blick auf die Möglichkeit der Befreiung von artenschutzrechtlichen Verboten $(\$ 67 \mathrm{BNatSchG)}$ und den Entschädigungsanspruch aus $\$ 68$ Abs. 1 BNatSchG gerichtet werden. Hierzu stellt das BVerwG klar, dass sowohl die Befreiung von naturschutzrechtlichen oder artenschutzrechtlichen Verboten aus $\$ 67$ Abs. 1, Abs. 2 S. 1 BNatSchG als auch der Entschädigungsanspruch aus $\$ 68$ Abs. 1 BNatSchG den Zweck verfolgen, ,die Verfassungsmäßigkeit naturschutzrechtlicher [oder artenschutzrechtlicher] Verbote im Einzelfall sicherzustellen und unverhältnismäßige Inhalts- und Schrankenbestimmungen des Eigentums, die der Gesetzgeber mit dem Begriff der (im Einzelfall) unzumutbaren Belastung erfasst, in erster Linie mit dem Instrument der Befreiung und nachrangig durch eine Entschädigung auszugleichen" ${ }^{2124}$. Daraus folgert das BVerwG, dass man den Begriff der ,unzumutbaren Belastung“" in $\$ 67$ Abs. 2 BNatSchG und $\$ 68$ Abs. 1 BNatSchG „,in demselben Sinne“ verstehen muss ${ }^{125}$.

$\mathrm{Zu}$ weiteren tatbestandlichen Voraussetzungen des $\$ 68$ Abs. $1 \mathrm{BNatSchG}$ hat das BVerwG festgestellt, dass der Begriff des „Eigentums“ in $\$ 68$ Abs. 1 BNatSchG alle durch Art. 14 GG geschützten Eigentumspositionen umfasst ${ }^{126}$, da $\$ 68$ Abs. 1 BNatSchG maßgeblich als verfassungskonforme Regelung zur Einschränkung des Eigentumsrechts aus Art. 14 GG anzusehen ist ${ }^{127}$. Aufgrund der Verzahnung von verfassungsrechtlichem Eigentumsbegriff und \68 Abs. 1 BNatSchG orientiert sich das Begriffsverständnis der „unzumutbaren Belastung“ maßgeblich daran, die verfassungsrechtlich wesentlich geschützte Privatnützigkeit des Grundeigentums bei der Anwendung der artenschutzrechtlichen Maßnahmen zu gewährleisten ${ }^{128}$. Als Maßstab definiert das BVerwG die Privatnützigkeit des Eigentums als ,absolute Grenze zumutbarer Nutzungseinschränkungen im Einzelfall“"129, die überschritten ist, wenn ,für ein Grundstück keinerlei sinnvolle Nutzung mehr besteht"130. Bei der Beurteilung der Eigentumsbeeinträchtigung spielen subjektive Umstände, insbesondere etwaige Vertrauensschutzgesichtspunkte, keine Rolle, da $\int 68$ Abs. 1 BNatSchG eine Verteilungsnorm darstellt, mit der „Nutzungsbeschränkungen im Bestand des Grundeigentums fi- nanziell ausgeglichen werden sollen." 131 Die Regelung sei aber nicht als Sanktionsnorm zu verstehen, bei der etwaiges subjektives Wissen eine Rolle spielen könnte ${ }^{132}$.

Der Tatbestand des $₫ 68$ Abs. 1 BNatSchG setzt außerdem voraus, dass artenschutzrechtliche Beschränkungen des Eigentums zu einer unzumutbaren Belastung „,führen“. Daraus lässt sich schließen, dass eine artenschutzrechtliche Beschränkung für die unzumutbare Belastung kausal sein muss, um einen Entschädigungsanspruch auszulösen ${ }^{133}$. Ist die Kausalität ,in tatsächlicher Hinsicht nicht mehr hinreichend sicher“ aufzuklären, muss ,eine Entscheidung nach Beweislastgrundsätzen“" erfolgen ${ }^{134}$. Schließlich kann eine Kausalität dann zu verneinen sein, wenn die geplante Nutzung des Grundstücks ,,aus anderen als naturschutzrechtlichen [oder artenschutzrechtlichen Gründen] ausgeschlossen war"“135.

\section{Resümee}

Trotz der vielen Facetten der dargestellten Judikate verdeutlicht die Analyse der Rechtsprechung zum Artenschutzrecht mehrerlei: Allein an der numerischen $\mathrm{Ku}-$ mulation von Urteilen zu bestimmten Themenbereichen lassen sich die „Brennpunkte“ des Artenschutzrechts zielsicher herauslesen: Die Verbotstatbestände des $\$ 44$ BNatSchG sind hier an vorderster Front zu finden. Außerdem steht das Artenschutzrecht Pate für eine verstärkt anzutreffende Erscheinung, wonach aus den feinsten Verästelungen des besonderen Verwaltungsrechts immer häufiger Impulse ausgehen, die das gesamte Verwaltungsrecht - wenn nicht gar das gesamte öffentliche Recht - signifikant beeinflussen können. Der Beschluss des BVerfG vom 23.10.2018 ${ }^{136}$ gehört sicherlich dazu. Nimmt man den dort festgestellten Handlungsauftrag an den Gesetzgeber ernst, in Bereichen unzureichender wissenschaftlicher Durchdringung von Wirkungszusammenhängen seiner ,,inhaltlichen Entscheidungsverantwortung“ gerecht zu werden und ,zumindest (zur) untergesetzliche(n) Maßstabsbildung" beizutragen, ${ }^{137}$

119) BVerwG, Urt. v. 23.4.2014 - 9 A 25.12, BVerwGE 149, 289 , Rdnr. 120.

120) BVerwG, Urt. v. 23.4.2014 - 9 A 25.12, BVerwGE 149, 289, Rdnr. 120.

121) BVerwG, Urt. v. 23.4.2014 - 9 A 25.12, BVerwGE 149, 289, Rdnr. 120.

122) BVerwG, Beschl. v. 31.7.2018 - 4 BN 13.18, Rdnr. 4.

123) BVerwG, Beschl. v. 31.7.2018 - 4 BN 13.18, Rdnr. 4.

124) BVerwG, Urt. v. 17.5.2018 - 4 C 2.17, BVerwGE 162, 127 , Rdnr. 20.

125) BVerwG, Urt. v. 17.5.2018 - 4 C 2.17, BVerwGE 162, 127, Rdnr. 20.

126) BVerwG, Urt. v. 25.10.2018 - 4 C 9.17, BVerwGE 163, 294, Rdnr. 24.

127) BVerwG, Urt. v. 17.5.2018 - 4 C 2.17, BVerwGE 162, 127, Rdnr. 12.

128) BVerwG, Urt. v. 25.10.2018 - 4 C 9.17, BVerwGE 163, 294, Rdnr. 30.

129) BVerwG, Urt. v. 25.10.2018 - 4 C 9.17, BVerwGE 163, 294, Rdnr. 29.

130) BVerwG, Urt. v. 25.10.2018 - 4 C 9.17, BVerwGE 163, 294, Rdnr. 29.

131) BVerwG, Urt. v. 25.10.2018 - 4 C 9.17, BVerwGE 162, 127 , Rdnr. 38

132) BVerwG, Urt. v. 25.10.2018 - 4 C 9.17, BVerwGE 162, 127, Rdnr. 38.

133) BVerwG, Urt. v. 25.10.2018 - 4 C 9.17, BVerwGE 162, 127, Rdnr. 46.

134) BVerwG, Urt. v. 25.10.2018 - 4 C 9.17, BVerwGE 162, 127, Rdnr. 48.

135) BVerwG, Urt. v. 25.10.2018 - 4 C 9.17, BVerwGE 162, 127 , Rdnr. 46.

136) BVerfG, Beschl. v. 23.10.2018 - 1 BvR 2523/13 und 1 BvR 595/14, BVerfGE 149, 407.

137) BVerfG, Beschl. v. 23.10.2018 - 1 BvR 2523/13 und 1 BvR 595/14, BVerfGE 149, 407, Rdnr. 24. 
werden sich hieran schnell grundsätzlich staatsrechtliche Folgefragen anschließen, deren Wirkung im gesamten öffentlichen Recht zu spüren sein werden. So darf man gespannt sein, unter welchen Voraussetzungen die höchsten Gerichte etwa von einem gesetzgeberischen Normierungsversagen ausgehen und welche Rechtsfolgen sie hieran knüpfen werden.

Open Access. Dieser Artikel wird unter der Creative Commons Namensnennung 4.0 International Lizenz veröffentlicht, welche die Nutzung, Vervielfältigung, Bearbeitung, Verbreitung und Wiedergabe in jeglichem Medium und Format erlaubt, sofern Sie den/die ursprünglichen Autor(en) und die Quelle ordnungsgemäß nennen, einen Link zur Creative Commons Lizenz beifügen und angeben, ob Änderungen vorgenommen wurden.

Die in diesem Artikel enthaltenen Bilder und sonstiges Drittmaterial unterliegen ebenfalls der genannten Creative Commons Lizenz, sofern sich aus der Abbildungslegende nichts anderes ergibt. Sofern das betreffende Material nicht unter der genannten Creative Commons Lizenz steht und die betreffende Handlung nicht nach gesetzlichen Vorschriften erlaubt ist, ist für die oben aufgeführten Weiterverwendungen des Materials die Einwilligung des jeweiligen Rechteinhabers einzuholen.

Weitere Details zur Lizenz entnehmen Sie bitte der Lizenzinformation auf http://creativecommons.org/licenses/by/4.0/deed.de.

Open access funding provided by Projekt DEAL.

\title{
Das Klimaschutzgesetz des Bundes - Hintergrund, Regelungsstruktur und wesentliche Inhalte
}

\author{
Juliane Albrecht*
}

(C) Der/die Autor(en) 2020

Das im Dezember 2019 in Kraft getretene Bundes-Klimaschutzgesetz (KSG) setzt in Deutschland erstmals einen dauerhaft verbindlichen Rahmen für den Klimaschutz und knüpft insofern an völker- und europarechtlichen Verpflichtungen an. Dieser Beitrag beschreibt den rechtlichen Hintergrund, die Regelungsstruktur und wesentliche Inhalte des Gesetzes. Dabei werden die einzelnen Vorschriften des KSG erläutert und mit den internationalen Vorgaben (Pariser Abkommen) sowie den aktuellen Instrumenten der EUKlimaschutzpolitik (Emissionshandels-RL, Klimaschutz-Verordnung, LULUCF-Verordnung und Governance-Verordnung) in Beziehung gesetzt sowie einer zusammenfassenden Bewertung unterzogen.

\section{Einführung}

Klimaschutz ist bereits seit längerem in der politischen und rechtlichen Diskussion. In etwa der Hälfte der Bundeländer liegen mittlerweile Klimaschutzgesetze vor. Seit dem Abkommen von Paris und „Fridays for Future“ ist das Thema mittlerweile auch auf Bundesebene ganz oben auf die politische Agenda gerückt. So hat die Bundesregierung im November 2016 den Klimaschutzplan 2050 als Langfriststrategie zum Pariser Übereinkommen vorgelegt. Am 18. Dezember 2019 ist nunmehr das Klimaschutzgesetz (KSG) in Kraft getreten. ${ }^{1}$ Damit wurde in Deutschland auf Bundesebene erstmals ein Klimaschutzgesetz mit verbindlichen Klimaschutzzielen eingeführt. Ziel des vorliegenden Beitrages ist es, Hintergrund, Regelungsstruktur die wesentlichen Inhalte des Gesetzes vorzustellen. Da die Vorgaben des KSG maßgeblich durch völker- und europarechtliche Anforderungen bestimmt sind, werden diese zunächst unter 2. näher beschrieben. Kap. 3 befasst sich mit der Entstehungsgeschichte des KSG, dessen Regelungsstruktur und wesentlichen Vorschriften. Der Beitrag schließt mit einer zusammenfassenden Bewertung der gesetzlichen Neuregelungen (Kap. 4).

Dr. Juliane Albrecht,

Leibniz-Institut für ökologische Raumentwicklung,

Dresden, Deutschland

\section{Völker- und europarechtliche Zusammenhänge}

Die Inhalte des neuen Bundesklimaschutzgesetzes erschließen sich maßgeblich vor dem Hintergrund völker- und europarechtlicher Vorgaben.

\subsection{Völkerrecht}

Grundlage des KSG ist das Übereinkommen von Paris (Paris Agreement, PA 2015) ${ }^{2}$, welches aufgrund der Klimarahmenkonvention der Vereinten Nationen (UNFCCC, 1992) ${ }^{3}$, abgeschlossen worden ist und das Kyoto-Protokoll ${ }^{4}$ ablöst. Die vormals schematische Zweiteilung in Industrieländer einerseits und Schwellen- und Entwicklungsländer andererseits mündet in einem multilateralen Klimaabkommen, welches sämtliche Vertragsstaaten, freilich unter Berücksichtigung ihrer Leistungsfähigkeit, verpflichtet. ${ }^{5}$ Art. 2 Abs. 1a PA enthält die Verpflichtung, den Anstieg der globalen Durchschnittstemperatur auf deutlich unter 2 Grad und möglichst auf 1,5 Grad gegenüber dem vorindustriellen Niveau zu begrenzen, um die Auswirkungen des weltweiten Klimawandels so gering wie möglich zu halten. Das Abkommen sieht kein genau definiertes Zeitziel für die Klimaschutzanstrengungen vor, sondern ist darauf ausgerichtet, in der zweiten Hälfte dieses Jahrhunderts ein Gleichgewicht zwischen den anthropogenen Emissionen von Treibhausgasen aus Quel-

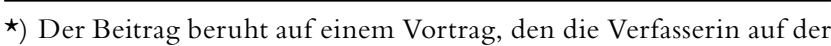
4. Bundesfachtagung Naturschutzrecht des Verbands Beruflicher Naturschutz (BBN) „Naturschutzrecht im Kontext von Klimawandel und Energiewende“ am 26.9.2019 an der Universität Kassel gehalten hat.

1) Bundes-Klimaschutzgesetz vom 12.12.2019 (BGB1. I S. 2513).

2) Paris Agreement, UNFCCC, Home, FCC/CP/2015/L.9/Rev.1 sowie BGBl. II 2016, 1082.

3) UN Framework Convention on Climate Change (UNFCCC), BGBl.1993 II 1784.

4) Kyoto Protocol to the United Nations Framework Convention on Climate Change, 1998; BGB1. II 2002, 966.

5) Saurer, NVwZ 2017, S. 1574; Schafhausen, in: Hebeler/Hofmann/ Proelß/Reif (Hrsg.), Die Zukunft der Energiewende, 2018, S. 11, 20. 